\title{
A new approach to bacterial colony morphotyping by matrix-assisted laser desorption ionization time of flight-based mass spectrometry
}

\author{
Ana Margarida Sousa ${ }^{a}$, J.D. Nunes-Miranda ${ }^{\text {b,c }}$, Miguel Reboiro-Jato ${ }^{\mathrm{d}}$, \\ Florentino Fdez-Riverola ${ }^{\mathrm{d}}$, Anália Lourenço ${ }^{\mathrm{a}, \mathrm{d}}$, Maria Olívia Pereira ${ }^{\mathrm{a}}$, J.L. Capelo ${ }^{\mathrm{b}, \mathrm{e}, *}$ \\ ${ }^{a}$ Institute for Biotechnology and Bioengineering, Centre of Biological Engineering, University of Minho, Campus de Gualtar, 4710-057 Braga, Portugal \\ ${ }^{\mathrm{b}}$ Bioscope Group, Physical Chemistry Department, Science Faculty, University of Vigo, Ourense, Spain \\ ${ }^{\mathrm{c}}$ Institute for Biotechnology and Bioengineering, Centre of Genomics and Biotechnology, University of Trás-os-Montes and Alto Douro, Vila Real, Portugal \\ ' SING Group, Informatics Department, Higher Technical School of Computer Engineering, University of Vigo, Ourense, Spain \\ e REQUIMTE, Departamento de Química, Faculdade de Ciencias e Tecnologia, FCT, Universidade Nova de Lisboa, 2829-516 Caparica, Portugal
}

\section{A R T I C L E I N F O}

\section{Article history:}

Received 4 December 2012

Received in revised form

21 April 2013

Accepted 24 April 2013

Available online 3 May 2013

Keywords:

Bacteria

MALDI

Morphotype

Classification

\begin{abstract}
A B S T R A C T
Matrix assisted laser desorption ionization time of flight mass spectrometry has been explored as a tool to bacterial colony morphotyping. To this end, four colony morphotypes of Pseudomonas aeruginosa and four of Staphylococcus aureus were analysed using intact bacteria. Results suggest that mass spectrometry of intact bacteria could, in some extent, be used to complement the classical morphological classification of bacteria.
\end{abstract}

(c) 2013 Elsevier B.V. All rights reserved.

\section{Introduction}

The fast and correct identification of infecting pathogens is the gold standard for successful, timely diagnosis and effective therapy design. To identify pathogens, there are several methods, which rely on culturing processes combined with morphological, physiological and biochemical characterization. Colony morphology observation often complements conventional microbial identification (e.g. biochemical tests) [1,2], namely to detect intra-strain diversity, i.e. to distinguish phenotypic subpopulations within the same strain $[3,4]$. For instance, the identification of $S$. aureus small colony variants (SCV) can be compromised since SCV often exhibit altered metabolic activity that influences the results displayed by biochemical tests [5,6].

Currently new technologies have emerged for fast and sensitive microbial identification, from which matrix-assisted laser desorption/ionization time of flight (MALDI-TOF) combined with mass spectrometry (MS) is the most promising one. MALDI-TOF MS has been extensively used for identification of bacteria at the species and strain level [7-12]. In fact, MALDI-TOF MS is now considered a

\footnotetext{
* Corresponding author at: Bioscope Group, Physical Chemistry Department, Science Faculty, As Lagoas, E-32004, Ourense, Spain. Tel.: +34 610835903 ; fax: +34988387001.

E-mail addresses: jlcapelom@uvigo.es, jlcm@fct.unl.pt (J.L. Capelo).
}

robust and reliable tool for bacterial taxonomy identification, as well as to classify bacterial species isolated from clinical samples [13]. Direct bacterial profiling by whole bacteria analysis is one of the most attractive approaches of MALDI-TOF MS, particularly in bacterial identification and differentiation. The direct deposition of "intact" bacteria mixed with matrix on the MALDI plate simplifies the laboratory procedures, thus minimising the costs and time taken in preparing the samples, without accuracy losses [14].

One of the major concerns of the medical community in terms of public health is the increasing resistance displayed by bacteria to conventional antimicrobials (disinfectants and antibiotics) $[15,16]$. Resistance seems to be potentiated when bacteria switch from planktonic or free-living state to sessile lifestyle and start growing as biofilms that are more resistant and persistent to antimicrobial stressors than their planktonic counterparts [17]. Several works have reported differences in antibiotic susceptibility profiles in colony morphologies isolated from biofilms [18-22] and, in particular, when bacteria are under antimicrobial stress after preventive or suppressive therapies [23,24]. In fact, a genetic homogenous population is able to introduce diversity in its population in order to ensure their survival against external stressors, such as antibiotics [25]. In addition to significant differences in resistance profiles to in-use antibiotics, further analysis of distinct morphotypes has revealed the expression of important virulence factors in infection and disease development 
as a function of the morphotype. Indeed, morphotypes identified as SCV [22,23] and mucoid variants [26,27] have been associated to highly resistance to some conventional antibiotics. Understanding such intra-strain diversity pointed out by colony morphology variation is crucial to design effective antibiotic therapies and minimise the occurrence of resistant or multi-resistant bacteria.

To date, MALDI-TOF MS ability to discriminate at colony morphology level has not been assessed. The present study aims to assess MALDI-TOF MS as a tool for colony morphology differentiation. To this end, a standardized sample preparation protocol for bacteria and a MS data analysis taken with a MALDI-based method elaborated for rapid colony morphology profiling was used as proof-of concept with two main human pathogenic bacteria, Pseudomonas aeruginosa and Staphylococcus aureus.

\section{Materials and methods}

\subsection{Materials and reagents}

All culture media used in this study were obtained from Liofilchem Diagnostic.

The following reagents were used to perform MALDI-TOF MS analysis: trifluoroacetic acid (TFA, 99\%) was from Riedel-de-Haen (Seelze, Germany), $\alpha$-cyano-4hydroxycinnamic acid ( $\alpha-\mathrm{CHCA}$ ) and sinapinic acid both puriss were from Fluka (Buchs, Switzerland). Milli-Q water was used throughout the experiments. All the chemicals used were of analytical-reagent grade, unless otherwise specified. All materials were used without further purification.

\subsection{Bacterial strains and culture conditions}

The strains tested were P. aeruginosa ATCC 10145 and S. aureus ATCC 25293. These bacteria were routinely cultured on tryptic soy broth (TSB) or agar (TSA) medium at $37^{\circ} \mathrm{C}$. All strains were preserved in criovials (Nalgene) at $-80 \pm 2{ }^{\circ} \mathrm{C}$. Prior to each experiment, bacterial cells were grown on solid media for $24 \mathrm{~h}$ at $37^{\circ} \mathrm{C}$.

\subsection{Biofilm formation}

P. aeruginosa ATCC 10145 and S. aureus ATCC 25293 were allowed to form 24-h-old biofilms as described previously [28]. Bacteria inocula were grown overnight on TSB at $37^{\circ} \mathrm{C}$ in air conditions, and then diluted in fresh TSB to obtain suspended cultures with $10^{7} \mathrm{CFU} / \mathrm{mL}$ as final concentration. Afterwards, each bacterial suspension was transferred to 6-well polystyrene plate, where biofilms were developed aerobically on a horizontal shaker $(120 \mathrm{rpm})$ at $37{ }^{\circ} \mathrm{C}$ for $24 \mathrm{~h}$. Then, biofilms were scrapped into sterile water, homogenized, being the biofilm-cells serially diluted with sterile ultrapure water and spread on solid media plates.

\subsection{Observation and classification of colony morphology}

To assess colony morphology, bacteria from planktonic and biofilm cultures were allowed to grow on solid plates during $50 \mathrm{~h}$ at $37^{\circ} \mathrm{C}$. This step was aimed to obtain complete and unchangeable morphotypes. Colony morphologies were observed by directly placing the petri plates on a magnifying glass (Olympus SZ-CTV) and photographed in a CCD camera (AVC, D5CE; Sony, Tokio, Japan). Colony morphotyping was based on a classification system of bacterial colonies, created by some of the authors within the scope of the community efforts promoted by the MIABiE consortium (http://miabie.org). Therefore, colonies were described using key morphological features commonly used in literature, including form, margin, surface, texture, size, presence of sheath, elevation, opacity, consistency and colour [29-31]. In addition, a quantitative parameter, the diameter, expressed in millimetres, was also included in the characterisation.

\subsection{Bacterial preparation for MALDI-TOF MS}

The different colonies of each bacterium were transferred from solid media to the extraction tube with a plastic loop. P. aeruginosa cells were re-suspended in ultrapure water and treated following a previous described protocol with some minor modifications as follows [32]. Cells were washed with $0.1 \% \mathrm{v} / \mathrm{v}$ of trifluoroacetic acid, TFA, re-suspended in $200 \mu \mathrm{L}$ of chloroform-methanol (1:1) and vortexed for $1 \mathrm{~min}$. The cell suspension was centrifuged at $9000 \mathrm{~g}$ for $8 \mathrm{~min}$ and the resulting pellet re-suspended in $15 \mu \mathrm{L}$ of $0.1 \% \mathrm{v} / \mathrm{v}$ of TFA. Different matrix solutions were daily prepared with $\alpha$-cyano-4-hydroxycinnamic acid ( $\alpha$-CHCA) and sinapinic acid as follows: $10 \mathrm{mg}$ of $\alpha$-CHCA were dissolved in 50\% acetonitrile $(\mathrm{ACN})$ and ultrapure water containing TFA $0.1 \% \mathrm{v} / \mathrm{v} ; 20 \mathrm{mg}$ of SA were dissolved in $30 \% \mathrm{ACN}$ and ultrapure water containing $0.3 \%$ of TFA. Then, each of the aforementioned matrices were 1:1 mixed with the cellular sample, and the mixture was vortexed for $30 \mathrm{~s}$. Each mixture (cell sample and matrix solution) was spotted on the MALDI target plate and allowed to dry at room temperature.

The treatment of $S$. aureus cells was performed according to Carbonnelle et al. [11] with some alterations. 1:1 v/v matrix to sample ratios were investigated by changing the matrix composition to assess the best matrix for this bacteria. The bacteria were first spotted into the MALDI plate and then the bacteria were covered by the matrix. The mixture was allowed to dry at room temperature.

\subsection{MALDI-TOF MS analysis}

An Ultraflex II MALDI-TOF/TOF instrument equipped with a nitrogen laser radiating at $337 \mathrm{~nm}$ from Bruker Daltonics was used to acquire bacteria mass spectra.

Measurements were done using the reflector positive ion mode, with a $20 \mathrm{kV}$ accelerating voltage, $75.1 \%$ grid voltage, $0.002 \%$ guide wire, and a delay time of $140 \mathrm{~ns}$. Mass spectra were taken in the $m / z$ range of $2000-20,000$ and obtained in different regions of the same sample, based on the acceptance criteria of 1000 laser shots per spot. External calibrations were performed with a Protein Calibration Standard I (Bruker Daltonics).

Mass spectra were processed with FlexAnalysis $\AA$ software (Version 2.4), subjected to baseline correction, noise filtration, normalized to the base peak, smoothed using Gauss algorithm and analysed considering the mass interval of 2000-20,000 Da (due to the good reproducibility of spectra profile at that range). In order to avoid the presence of noisy peaks, each sample was spotted five times in the MALDI MS, the resulting spectra were aligned allowing an $\mathrm{m} / \mathrm{z}$ error of $750 \mathrm{ppm}$, and those peaks that did not appear in, at least, three of the five spectra were discarded.

\subsection{Clustering analysis}

An agglomerative hierarchical clustering was employed to analyze the individual spectrum and peak similarities. The clustering was constructed using a custom implementation of the Unweighted Pair Group Method with Arithmetic Mean [33] algorithm with Hamming distance [34] as the distance metric. It was decided to choose this distance metric instead of the more common Euclidean distance since it was not used the peak intensity information. Therefore, the clustering was built taking into account solely the presence or the absence of the peaks in each spectrum. The clustering results were presented as heat maps using the Java Treeview software [35]. 
The peak lists used in the clustering analysis were filtered applying a Chi-squared test of independence corrected using Williams' Correction, and selecting those peaks with a $p$-value under 0.05 . That is, only the most discriminative peaks were used. Full list of peaks is provided in supplementary material (the peaklist.tvalues.xlsx file).

\section{Results and discussion}

\subsection{MALDI-TOF MS analysis optimization}

A total of 4 morphotypes for each species were studied. And for each morphotype a total of three to six biological replicates were used. The range of replicates was variable because of distinct prevalence of each morphotype in planktonic and biofilm cultures. Each biological replicate was spotted into the MALDI plate five times.

To clean bacteria from contaminants originated by the culture medium that would eventually cause interfering $m / z$ peaks in the MALDI spectrum, a solution of chloroform and methanol was used. This solution has been addressed in literature to remove culture residues from the surface of the bacteria [32]. After the cleaning procedure, the bacteria were pelleted by centrifugation. Then the pellet was resuspended in $0.1 \% \mathrm{v} / \mathrm{v}$ TFA. Before MALDI analysis, an equal volume of sample and matrix, $\alpha$-CHCA or sinapinic acid, was mixed. The mixture was then vortexed and $1 \mu \mathrm{L}$ of this solution was spotted into the MALDI target in five different spots.

\subsubsection{Influence of the type of matrix}

The matrix used to aid in the ionization process is of main concern in MALDI analysis. Thus, $\alpha$-CHCA is used in those cases where the analytes are mainly peptides with molecular masses below $10 \mathrm{KDa}$, whilst sinapinic acid is used for those cases where the analytes are high mass peptides or proteins, above $10 \mathrm{KDa}$ [36]. Fig. 1 shows spectra of different morphotypes of $P$. aeruginosa using both matrixes. From this figure it may be concluded that the spectrum obtained with $\alpha$-CHCA have more $\mathrm{m} / \mathrm{z}$ signals with higher intensity that the ones observed in the spectrum obtained with sinapinic acid. In other words the best matrix for $P$. aeruginosa was anticipated to be $\alpha-C H C A$, which was later confirmed by statistical assay (data not shown). The richest is the spectra in $\mathrm{m} / \mathrm{z}$ signals the best is the classification attained, as there is a higher chance to find differences between morphotypes.

Interestingly, the method mentioned above provided excellent spectra for the $P$. aeruginosa, but failed to obtain $\mathrm{m} / \mathrm{z}$ signals for S. aureus (data not shown). It was hypothesised that this was due to the low mass of bacteria transferred to the MALDI plate and differences in the size of both types of bacteria. Furthermore, colonies in this study were allowed to grow during $72 \mathrm{~h}$ to achieve complete colony morphogenesis, which may have affected the obtainment of MALDI-TOF MS spectra. It has been reported that excessive cultivation time (colony growth above $48 \mathrm{~h}$ ) could weak and reduce the detection of characteristic peaks by MALDITOF MS [37].

Consequently, for the case of $S$. aureus a different approach was attempted. First, the cleaning step was avoided, as it was observed that some bacteria were lost during this step. However, again no signal was obtained. So, an additional change was made.
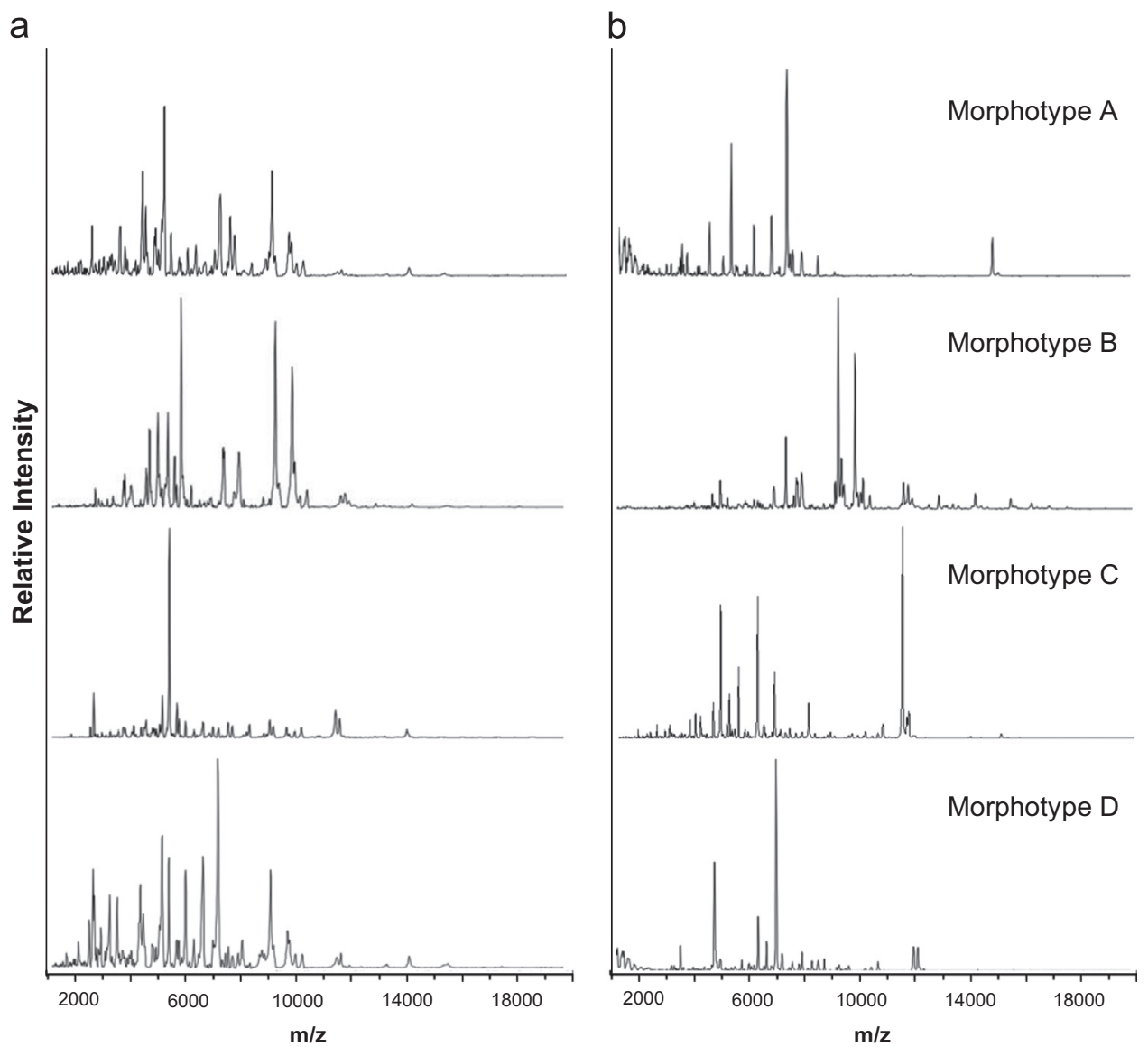

Fig. 1. MALDI-TOF MS spectra of the different colony morphotypes of $P$. aeruginosa obtained using (a) $\alpha$-CHCA and (b) sinapinic acid matrices. 
The crystallization process was modified. It is well known that in many cases the crystallization process severely affects the MALDI spectrum [36]. In this case, first the bacterial solution was spotted and let to dry. Then, the matrix solution ( $\alpha$-CHCA or sinapinic acid) was spotted over the dry sample. Once the matrix was also dry, the MALDI analysis was performed. With such modifications, good spectra were obtained using either $\alpha$-CHCA or sinapinic acid, as shown in Fig. 2. As a matter of fact, the spectra were similar for both $\alpha$-CHCA and sinapinic acid, being difficult to distinguish them by naked eye. So, for this case it was not possible to anticipate which matrix would provide the best results for classification purposes.

\subsection{Colony morphology classification}

Although colony morphology studies are attracting attention, the identification and description of morphological features are often inconsistent and somewhat confusing, relying on considerations of individual author and laboratories [38,39]. For instance, authors often use "wrinkled" and "rugose" as synonyms, but there are works in which that is not the case $[20,22]$. In this work, a new standardising morphological classification system was at the base of the colony annotation process (http://www.miabie.org/cmo. php). Morphological criteria used in this work include form, margin, surface, texture, size, presence of sheath, elevation, opacity, consistency and colour. Based on the aforementioned criteria four morphotypes of $P$. aeruginosa and four of $S$. aureus were classified as described in Table 1 . As may be seen, in terms of morphology, $P$. aeruginosa morphotype A differs from B, C and D in 3 of the 10 parameters investigated. B differs from $C$ and $D$ in 2 parameters, and $C$ differs from $D$ on one parameter. In other words, the classification seems as follows $A \gg B, C, D$; $B \gg C, D$; and $\mathrm{C}>\mathrm{D}$, where each " $>$ " means one parameter of difference.

For the case of $S$. aureus, the morphological classification was more troublesome. Non- and SCV morphotypes only differed in a maximum of two parameters (Table 1). Thus, morphotypes A and $B$ differ in two parameters as well as B and C. A and B differ from C and $D$ in one parameter whilst $C$ and $D$ differ between them in one parameter. In other words, $A \gg ; B ; B \gg C ; A>C, D ; B>D ; C>D$.

In an attempt to classify those morphotypes in a new, fast and straightforward way, we used MALDI mass spectrometry-based fingerprinting, as described below.

\subsection{MALDI-TOF MS classification}

The spectra obtained, a total of 140 , were clustered using the methodology described in Section 2.7. For the P. aeruginosa, a cluster was done with the data taken from the spectra that were obtained using $\alpha$-CHCA. This cluster is depicted in Fig. 3 . As may be seen, MALDI clusters the morphotypes into three main groups, two single groups constituted by the morphotypes $C$ and $D$ and a group constituted by morphotypes A and B. Morphotype D is clearly separated from the other morphotypes, while morphotype $C$ appears slightly mixed with some samples of morphotype B. Although samples of morphotype A are also mixed with samples of morphotype $B$, there is a clear separation between morphotype $A$ and morphotypes $C$ and $D$. The same result was attained with the spectra obtained with sinapinic acid.

The characteristic peaks concerning the three groups (A/B, C and $D$ ) were compared with those reported in literature. It was found that this type of information is not abundant because the studies regarding species identification by MALDI-TOF MS are focused on specific parameters, such as the matching at genus

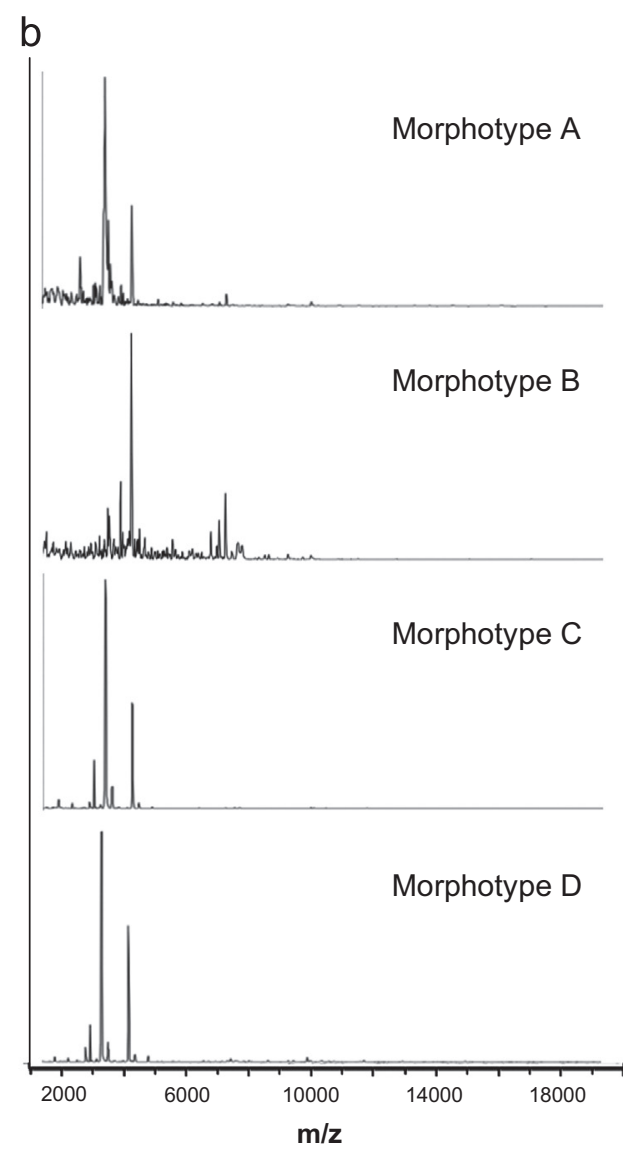

Fig. 2. MALDI-TOF MS spectra of the different colony morphotypes of S. aureus obtained using (a) $\alpha$-CHCA and (b) sinapinic acid matrices. 
Table 1

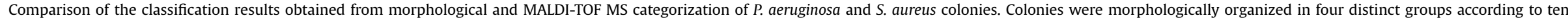
qualitative and one quantitative criteria. In contrast MALDI-TOF MS grouped colony morphotypes in two major groups (A and B; C and D) for P. aeruginosa and in two groups (A and B; C and D) for S. aureus.

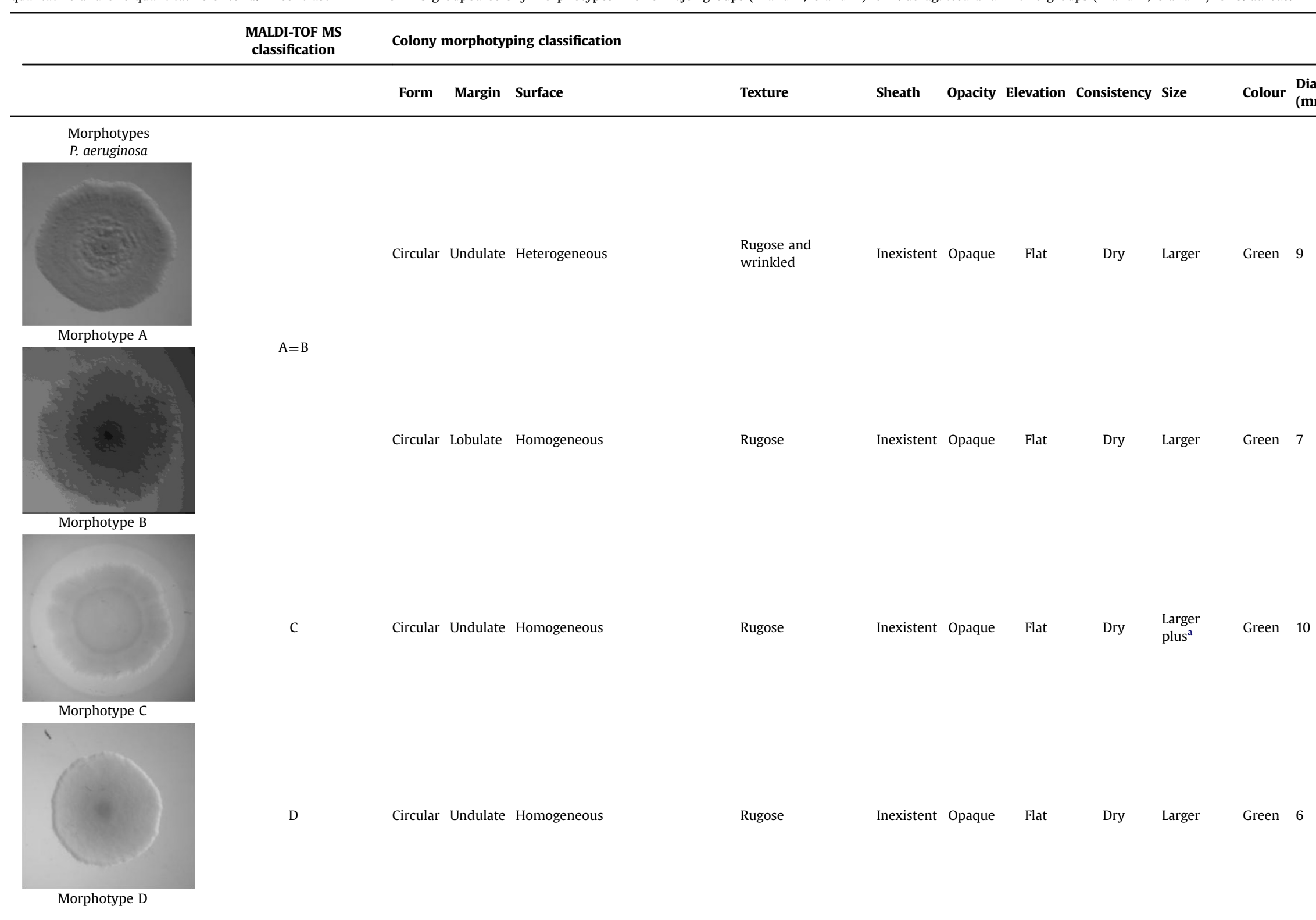




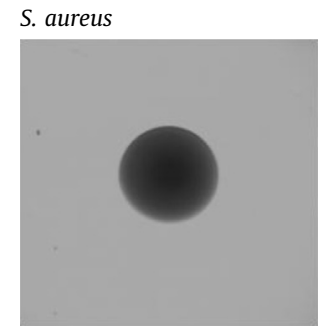

Morphotype A

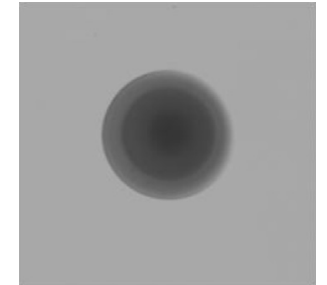

Morphotype B

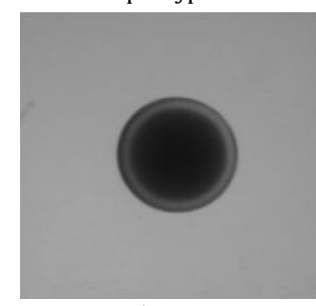

Morphotype C

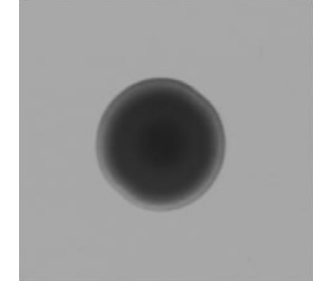

Morphotype D
Circular Entire

Homogeneous

Homogeneous with 3 distinc zones

Homogeneous with 2 distinct

Circular Entire

Circular Entir$$
\text { zones }
$$

Smooth

Inexistent Opaque Flat

Dry

Yellow 3
Homogeneous with 3 distinct zones
Inexistent Opaque Flat

Smooth

Inexistent Opaque Flat

Dry

Small

Yellow 2

Circular Entire
Smooth
Inexistent Opaque Flat

${ }^{\text {a }}$ The designation of larger plus is attributed to colonies with diameters equal and above $10 \mathrm{~mm}$ 




Fig. 3. Heat map for the clustering of the $P$. aeruginosa spectra data generated using $\alpha$-CHCA matrix. Vertical dendrogram shows the samples hierarchical clustering, while the horizontal dendrogram shows the $m / z$ signals hierarchical clustering.

and species level and non-matching percentages $[1,12,40]$. Furthermore, peaks reported by those studies correspond to conserved domains expressed by the whole species, which do not apply to the present study. No common peaks were found between the list of characteristic peaks reported by strain differentiation studies by MALDI-TOF MS and the list of peaks reported by this study. Typically, peaks obtained from strain differentiation studies are characteristic of each bacterial strain, whilst the present study was focused on intra-strain level differentiation.

For S. aureus, the cluster done with the data taken from $\alpha-\mathrm{CHCA}$ which is shown in Fig. 4 reveals two main groups, one constituted by morphotypes A and B and another one formed by $C$ and D. Although samples of morphotype $D$ are grouped together, the number of peaks shared with samples of morphotype $C$ indicates that both morphotypes are very similar. This group is more homogeneous than the groups $A$ and $B$, where two samples (i.e. A_3_314 and B_2_60) were placed closer to the groups C and D than to their corresponding group. This classification was also obtained with sinapinic acid although was better attained with the $\alpha-\mathrm{CHCA}$, because for the case of sinapinic acid one A and one B morphotypes were mixed with the morphotypes $C$ and $D$.

The comparison of the characteristic peaks obtained for the two $S$. aureus groups with those reported in literature revealed that the 2646 of $\mathrm{m} / \mathrm{z}$ signal was already noticed. Edward-Jones et al. [41] pointed out a peak of $2647 \mathrm{~m} / \mathrm{z}$ value as one of the characteristic peaks exhibited by methicillin-sensitive staphylococcal isolates. Such evidence suggests that C and D morphotypeassociated bacteria may be susceptible to methicillin, in contrast to morphotypes A and B.

\subsection{MALDI-TOF MS versus morphological classification of bacterial colonies}

The classifications done over P. aeruginosa and $S$. aureus by the classic and the MALDI methods are shown in Table 1. For the case of $P$. aeruginosa, the morphological classification of colonies

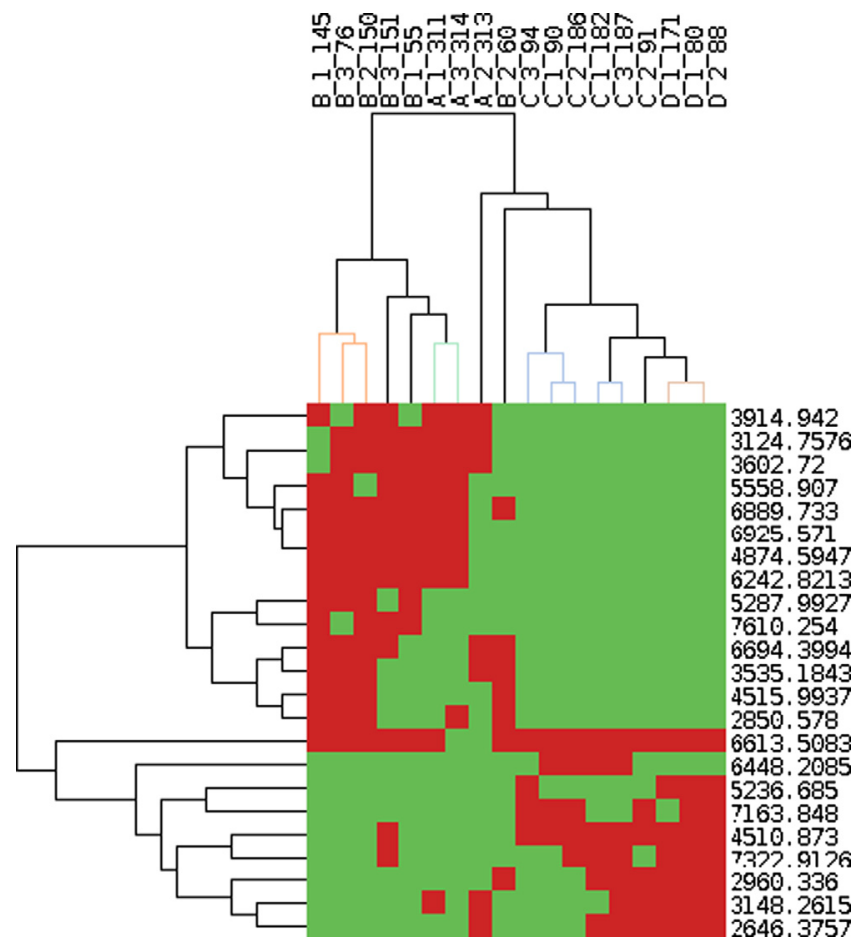

Fig. 4. Heat map for the clustering of the $S$. aureus spectra data generated using $\alpha$-CHCA matrix. Vertical dendrogram shows the samples hierarchical clustering, while the horizontal dendrogram shows the $m / z$ signals hierarchical clustering.

provides 4 phenotypes whilst the MALDI classifies only into three phenotypes because morphotypes A and B seemed similar in terms of their MALDI fingerprintings. This group is different from the other two groups formed by C and D.

The morphological classification done for $S$. aureus gave rise to four phenotypes whilst the clustering done with the MALDI spectra classified the morphotypes into two groups: A, B and C, D. It should be noted that on the first group MALDI-TOF MS analysis clustered a non- and SCV morphotypes, while the second group was composed only of non-SCV morphotypes.

Possibly, the discrepancies observed between MALDI-TOF MS and colony morphology methods for P. aeruginosa and S. aureus may arise due to the differential antibiotic patterns exhibited by the colony morphotype-associated bacteria. Indeed, a number of studies have reported that MALDI-TOF MS may distinguish antibiotic resistant and susceptible strains of the same species [41-45]. In addition, the expression of differential virulence factors may also contribute to discrepancies between both techniques. These hypotheses will be addressed in future works.

\section{Conclusions}

As a general trend seems that MALDI-based fingerprinting of bacterial colony variants is not able to provide a classification as large as the one obtained with the morphological classification using ten different visual characteristics. The distinct colony morphotypes were grouped by the MALDI-based strategy differently than using the morphological approach. However, the MALDI spectra can be also used as a further characteristic in helping to differentiate morphotypes. Taken together, these data suggest that MALDI-based morphotyping offers a different perspective than the classic morphological approach, which needs to be further explored. Future work will encompass correlating morphotyping of bacteria by both methodologies with antibiotic susceptibility and virulence factors expression. 


\section{Acknowledgments}

Financial support from IBB-CEB and Fundação para a Ciência e Tecnologia (FCT) and European Community fund FEDER, through Program COMPETE, in the ambit of the FCT project "PTDC/SAUSAP/113196/2009/ FCOMP-01-0124-FEDER-016012" and Ana Margarida Sousa PhD grant (SFRH/BD/72551/2010), is gratefully acknowledged. Authors also thank Portugal-Spain cooperation action sponsored by the Foundation of Portuguese Universities [E 48/11] and the Spanish Ministry of Science and Innovation [AIB2010PT-00353].

\section{Appendix A. Supporting information}

Supplementary data associated with this article can be found in the online version at http://dx.doi.org/10.1016/j.talanta.2013.04.058.

\section{References}

[1] S.Y. Hsieh, C.L. Tseng, Y.S. Lee, A.J. Kuo, C.F. Sun, Y.H. Lin, J.K. Chen, Mol Cell Proteomics 7 (2008) 448-456.

[2] S. Qamer, J.A. Sandoe, K.G. Kerr, J. Clin. Microbiol. 41 (2003) 2644-2646.

[3] D. Hilmi, M. Parcina, K. Bode, J. Ostrop, S. Schuett, K. Heeg, W. Ziebuhr, O. Sommerburg, I. Bekeredjian-Ding, Int. J. Med. Microbiol. 303 (2013) 61-69.

[4] C. Goerke, C. Wolz, Int. J. Med. Microbiol. 300 (2011) 520-525.

[5] R.A. Proctor, P. van Langevelde, M. Kristjansson, J.N. Maslow, R.D. Arbeit, Clin. Infect. Dis. 20 (1995) 95-102.

[6] R.A. Proctor, C. von Eiff, B.C. Kahl, K. Becker, P. McNamara, M. Herrmann, G. Peters, Nature Rev. Microbiol. 4 (2006) 295-305.

[7] E.N. Ilina, A.D. Borovskaya, M.M. Malakhova, V.A. Vereshchagin, A.A. Kubanova, A.N. Kruglov, T.S. Svistunova, A.O. Gazarian, T. Maier, M. Kostrzewa, V.M. Govorun, J. Mol. Diagn. 11 (2009) 75-86.

[8] L.G. Stevenson, S.K. Drake, P.R. Murray, J. Clin. Microbiol. 48 (2010) 444-447.

[9] M.F. Mazzeo, A. Sorrentino, M. Gaita, G. Cacace, M. Di Stasio, A. Facchiano, G. Comi, A. Malorni, R.A. Siciliano, Appl. Environ. Microb. 72 (2006) 1180-1189.

[10] Y.M. Williamson, H. Moura, A.R. Woolfitt, J.L. Pirkle, J.R. Barr, G. Carvalho Mda, E.P. Ades, G.M. Carlone, J.S. Sampson, Appl. Environ. Microb. 74 (2008) 5891-5897.

[11] E. Carbonnelle, J.L. Beretti, S. Cottyn, G. Quesne, P. Berche, X. Nassif, A. Ferroni, J. Clin. Microbiol. 45 (2007) 2156-2161.

[12] K. Sogawa, M. Watanabe, K. Sato, S. Segawa, C. Ishii, A. Miyabe, S. Murata T. Saito, F. Nomura, Anal. Bioanal. Chem. 400 (2011) 1905-1911.

[13] 〈http://www.bruker.com/en/products/mass-spectrometry-and-separations/ maldi-biotyper/overview.html $\rangle$, [accessed 21.04.13].

[14] M. Welker, E.R. Moore, Syst. Appl. Microbiol. 34 (2011) 2-11.

[15] P.M. Hawkey, J. Antimicrob. Chemother. 62 (Suppl. 1) (2008) i1-9.
[16] K. Smith, I.S. Hunter, J. Med. Microbiol. 57 (2008) 966-973.

[17] Y.A. Nikolaev, V.K. Plakunov, Microbiology 76 (2007) 125-138.

[18] E. Drenkard, F.M. Ausubel, Nature 416 (2002) 740-743.

[19] M. Allegrucci, K. Sauer., J. Bacteriol. 189 (2007) 2030-2038.

[20] M.J. Kirisits, L. Prost, M. Starkey, M.R. Parsek., Appl. Environ. Microb. 71 (2005) 4809-4821.

[21] I.R. Monk, G.M. Cook, B.C. Monk, P.J. Bremer., Appl. Environ. Microb. 70 (2004) 6686-6694.

[22] M. Starkey, J.H. Hickman, L. Ma, N. Zhang, S. De Long, A. Hinz, S. Palacios, C. Manoil, M.J. Kirisits, T.D. Starner, D.J. Wozniak, C.S. Harwood, M.R. Parsek, J. Bacteriol. 191 (2009) 3492-3503.

[23] R. Singh, P. Ray, A. Das, M. Sharma, J. Med. Microbiol. 58 (2009) 1067-1073.

[24] J.B. Lyczak, C.L. Cannon, G.B. Pier, Clin. Microbiol. Rev. 15 (2002) 194-222.

[25] A.M. Sousa, I. Machado, M.O. Pereira, Phenotypic switching: an opportunity to bacteria thrive, in: A. Mendez-Vilas (Ed.) Science Against Microbial Pathogens: Communicating Current Research and Technological Advances, 2011.

[26] S.M. Kirov, J.S. Webb, Y. O'May, C.D.W. Reid, J.K. Woo, S.A. Rice, S. Kjelleberg, Microbiology 153 (2007) 3264-3274.

[27] J.R. Govan, V. Deretic., Microbiol. Rev. 60 (1996) 539-574.

[28] S. Stepanovic, D. Vukovic, I. Dakic, B. Savic, M. Svabic-Vlahovic, J. Microbiol. Meth. 40 (2000) 175-179.

[29] S.K. Hansen, M.H. Rau, H.K. Johansen, O. Ciofu, L. Jelsbak, L. Yang, A. Folkesson, H.O. Jarmer, K. Aanaes, C. von Buchwald, N. Hoiby, S. Molin., ISME J. 6 (2012) 31-45.

[30] Y.S. Chen, H.H. Lin, C.C. Hung, J.J. Mu, Y.S. Hsiao, Y.L. Chen, Microbiol. Immunol. 53 (2009) 184-189.

[31] N. Chantratita, V. Wuthiekanun, K. Boonbumrung, R. Tiyawisutsri, M. Vesaratchavest, D. Limmathurotsakul, W. Chierakul, S. Wongratanacheewin, S. Pukritiyakamee, N.J. White, N.P. Day, S.J. Peacock., J. Bact. 189 (2007) 807-817.

[32] H. Liu, Z. Du, J. Wang, R. Yang, Appl. Environ. Microb. 73 (2007) 1899-1907.

[33] R.R. Sokal, C.D. Michener, University of Kansas Scientific Bulletin 28 (1958) 1409-1438.

[34] R.W. Hamming, Bell Syst. Tech. J. 29 (2) (1950) 147-160.

[35] A.J. Saldanha, Bioinformatics 20 (2004) 3246-3248.

[36] F. Hillenkamp, M. Karas, The MALDI Process and Method, in: M.S. MALDI (Ed.), A Practical Guide to Instrumentation, Methods and Applications, Wiley-VCH Verlag GmbH \& Co. KGaA, 2007, pp. 1-28.

[37] A. Wieser, L. Schneider, J. Jung, S. Schubert, Appl. Microbiol. Biotechnol. 93 (2012) 965-974.

[38] A.M. Sousa, A. Ferreira, N.F. Azevedo, M.O. Pereira, A. Lourenco, J. Integr., Bioinform 9 (2012) 203.

[39] A.M. Sousa, A. Lourenço, M.O. Pereira, MorphoCol: a powerful tool for the clinical profiling of pathogenic bacteria, Advances in Intelligent and Soft Computing, Springer181-187.

[40] U. Eigner, M. Holfelder, K. Oberdorfer, U. Betz-Wild, D. Bertsch, A.M. Fahr, Clin. Lab. 55 (2009) 289-296.

[41] V. Edwards-Jones, M.A. Claydon, D.J. Evason, J. Walker, A.J. Fox, D.B. Gordon, J. Med. Microbiol. 49 (2000) 295-300.

[42] P. Seng, M. Drancourt, F. Gouriet, B. La Scola, P.E. Fournier, J.M. Rolain, D. Raoult, Clin. Infect. Dis. 49 (2009) 543-551.

[43] M. Wolters, H. Rohde, T. Maier, C. Belmar-Campos, G. Franke, S. Scherpe, M. Aepfelbacher, M. Christner, J. Int, Med. Microbiol. 301 (2011) 64-68.

[44] J.E. Camara, F.A. Hays, Anal. Bioanal. Chem. 389 (2007) 1633-1638.

[45] Z. Du, R. Yang, Z. Guo, Y. Song, J. Wang, Anal. Chem. 74 (2002) 5487-5491. 